cAMP production mediated by the Arg389 variant or whether antagonism of these other receptors is involved in the beneficial effects of carvedilol in HF. However, what is important to remember based on the current work is that for the $\beta_{1}$-AR, carvedilol displays the greatest degree of inverse agonism, especially for the Arg389 variant (Figure 1), and studies such as this could lead to research broadening our knowledge of this pharmacological property of GPCR ligands and therefore potentially increase their clinical utility (16).

\section{Taking it personally}

The implications of these findings are of potentially profound clinical importance when considering the interindividual and ethnic variation that occurs in response to $\beta$-AR blocker therapy in the treatment of HF. It has been reported that the allele frequency of the Arg389 variant is $20 \%$ less common in black patients compared with non-black patients, and this may partially explain the poorer response to $\beta_{1}$-AR antagonists seen in blacks compared with that of the rest of the population (17). Future efforts to characterize the effect of genetic heterogeneity in cellular response to pharmacological agents, such as the $\beta_{1}$-AR antagonists examined in this study, lend credence to the viability of the concept of pharmacogenomics - pharmacologic intervention adapted according to an individual's genetic makeup - and the realization of "personalized" medicine for the treatment of cardiovascular disorders such as HF (18).

Address correspondence to: Walter J. Koch, Center for Translational Medicine, George Zallie and Family Laboratory for Cardiovascular Gene Therapy, Department of Medicine, Thomas Jefferson University, 1025 Walnut Street, Room 317, Philadelphia, Pennsylvania 19107, USA. Phone: (215) 955-9982; Fax: (215) 503-5731; E-mail: walter.koch@jefferson.edu.

1. Lee, T.H. 2001. Management of heart failure: AHA/ ACC guidelines summary. Heart disease: a textbook of cardiovascular medicine. 6th edition. E. Braunwald, editor. 101:652-685.

2. Becker, O.M., et al. 2004. G-protein coupled receptors: in silico drug discovery in 3D. Proc. Natl. Acad. Sci. U. S. A. 101:11304-11309.

3. Rockman, H.A., Koch, W.J., and Lefkowitz, R.J. 2002. Seven-transmembrane-spanning receptors and heart function. Nature 415:206-212.

4. Maqbool, A., Hall, S.A., Ball, S.G., and Balmforth, A.J. 1999. Common polymorphisms of $\beta_{1}$-adrenoreceptor: identification and rapid screening assay [research letter]. Lancet. 353:897.

5. Ranade, K., et al. 2002. A polymorphism in the $\beta_{1}$ adrenergic receptor is associated with resting heart rate. Am. J. Hum. Genet. 70:935-942.

6. Small, K.M., McGraw, D.W., and Liggett, S.B. 2003 Pharmacology and physiology of human adrenergic receptor polymorphisms. Annu. Rev. Pharmacol. Toxicol. 43:381-411

7. Mason, D.A., Moore, J.D., Green, S.A., and Liggett, S.B. 1999. A gain-of-function polymorphism in a Gprotein coupling domain of the human $\beta_{1}$-adrenergic receptor. J. Biol. Chem. 274:12670-12674.
8. Akhter, S.A., D’Souza, K.M., Petrashevskaya, N.N., Mialet-Perez, J., and Liggett, S.B. 2006. Myocardial $\beta_{1}$-adrenergic receptor polymorphisms affect functional recovery after ischemic injury. Am. J. Physiol. Heart Circ. Physiol. 290: H1427-H1432.

9. Brauwald, E., and Bristow, M.R. 2002. Congestive heart failure: fifty years of progress. Circulation. 102(20 Suppl. 4):IV14-IV23.

10. Beta-blocker Evaluation of Survival Trial Investigators. 2001. A trial of the beta-blocker bucindolol in patients with advanced chronic heart failure. N. Engl. J. Med. 344:1659-1667.

11. Leinweber, K. 2004. Beta-adrenergic receptor polymorphism in human cardiovascular disease. Ann. Med. 36:64-69.

12. Giempmans, B.N., Adams, S.R., Ellisman, M.H., and Tsien, R.Y. 2006. The fluorescent toolbox for assessing protein localization and function. Science. 312:217-224.

13. Miyawaki, A. 2003. Visualization of the spatial and temporal dynamics of intracellular signaling. Dev. Cell. 4:295-305.

14. Rochais, F., et al. 2007. Real-time optical recording of $\beta_{1}$-adrenergic receptor activation reveals supersensitivity of the Arg389 variant to carvedilol. J. Clin. Invest. 117:229-235. doi:10.1172/JCI30012.

15. Yancy, C.W., et al. 2001. Race and the response to adrenergic blockade with carvedilol in patients with chronic heart failure. N. Engl. J. Med. 344:1358-1364.

16. Bond, R.A., and Ijzerman, A.P. 2006. Recent developments in constitutive receptor activity and inverse agonism, and their potential for GPCR drug discovery. Trends Pharmacol. Sci. 27:92-96.

17. Small, K.M., Wagoner, L.E., Levin, A.M., Kardia, S.L., and Liggett, S.B. 2002. Synergistic polymorphisms of $\beta_{1^{-}}$and $\alpha_{2 \mathrm{c}}$-adrenergic receptors and the risk of congestive heart failure. N. Engl. J. Med. 347:1135-1142.

18. Zineh, I., and Johnson, J.A. 2006. Pharmacogenetics of chronic cardiovascular drugs: applications and implications. Expert Opin. Pharmacother. 11:1417-1427.

\title{
Macrophage heterogeneity and tissue lipids
}

\author{
Siamon Gordon
}

Sir William Dunn School of Pathology, University of Oxford, Oxford, United Kingdom.

\begin{abstract}
Macrophages are present as resident cells in adipose tissue, and blood monocytes are recruited in increased numbers to sites of lipid accumulation in atherosclerosis, a modified form of inflammation in the arterial wall. Recent findings reported by 3 separate groups in this issue of the JCI provide evidence for distinct monocyte subsets, differential chemokine receptor usage, and phenotypic modulation of macrophages in murine models of genetic and high-fat diet-induced disease (see the related articles beginning on pages 175, 185, and 195). These studies raise prospects for selective therapeutic targets to ameliorate macrophage hyperinflammatory responses, while sparing host defense and repair mechanisms.
\end{abstract}

Nonstandard abbreviations used: CCR2, C-C motif chemokine receptor 2; CX3CR1, C- $\mathrm{X}_{3}-\mathrm{C}$ motif chemokine receptor 1 .

Conflict of interest: The author has declared that no conflict of interest exists.

Citation for this article: J. Clin. Invest. 117:89-93 (2007). doi:10.1172/JCI30992.
Monocytes and mature macrophages are prominent in the host response to lipid accumulation in major arteries, the development of atherosclerotic plaques, and their complications (1). Less established are the details of their colocalization and possible metabolic interactions with adi- pocytes in body fat stores (2). New studies reported in this issue of the JCI by Swirski et al. (3) demonstrate that a monocyte subset that expresses high levels of a marker antigen, Ly-6C, dominates hypercholesterolemia-associated monocytosis and gives rise to macrophages in atheromata. Also in this issue, Tacke et al. (4) report that monocyte subsets differentially employ the chemokine receptors $\mathrm{C}-\mathrm{C}$ motif chemokine receptor 2 (CCR2), CCR5, and C- $\mathrm{X}_{3}-\mathrm{C}$ motif chemokine receptor 1 (CX3CR1, also known as the fractalkine receptor) to enter atherosclerotic plaques. They also exploited an mAb, Gr-1, directed against Ly- 6 family antigens to distinguish monocyte subsets and used CD11c, a $\beta 2$ integrin expressed by myeloid DCs and selected tis- 


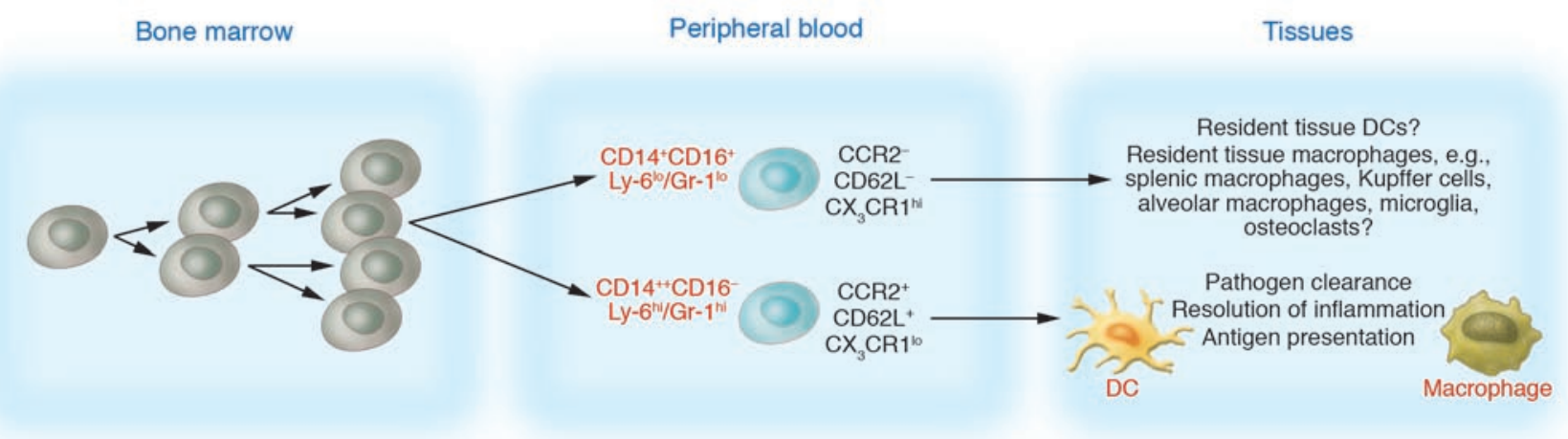

\section{Figure 1}

Heterogeneity of monocytes and tissue macrophages. This schematic summarizes previous evidence for monocytic subsets giving rise to tissue macrophages and myeloid DCs in the absence and presence of microbially elicited inflammation. Two distinct subpopulations of monocytes can

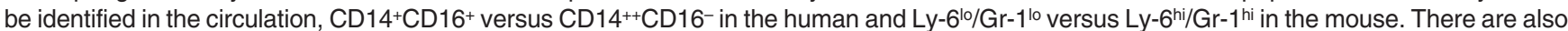
differences in expression of CX3CR1 (fractalkine receptor), CCR2, and CD62L ( $L$ selectin ligand). Experimental studies in rodents have shown that the CX3CR $1^{\text {lo }}$ subset gives rise to recruited macrophages and DCs, in response to a proinflammatory stimulus, whereas the CX3CR $1^{10}$ subset may give rise to resident tissue cells.

sue macrophages, to characterize the phenotype of macrophage subpopulations in lesions. In the third of this trio of articles, Lumeng et al. (5), by contrast, document that adipose tissue macrophages, which accumulate with obesity and are implicated in insulin resistance, switch their phenotype from one of an alternatively activated (M2) polarization to proinflammatory (M1) cells and that a subset of these cells expresses elevated levels of the CD11c marker. Collectively, these studies extend our knowledge of heterogeneity of monocyte recruitment and the macrophage phenotype in response to different inflammatory and metabolic stimuli and reinforce the importance of macrophage interactions with adipocytes, as well as with endothelium, smooth muscle, and other cells in the lipid-rich tissue microenvironment.

\section{Monocyte/macrophage heterogeneity} Lipid-laden macrophages, known as foam cells, play a central role in the pathogenesis and repair of atherosclerotic plaques, the product of local cellular inflammatory reactions in arterial walls (6). Macrophages have also been implicated in plaque vulnerability to rupture and thrombus formation. Their recruitment depends on circulating monocyte levels and is greatly reduced in M-CSF-deficient osteopetrotic mice, which display a variable deficiency of tissue macrophage populations in the absence of this important growth and survival factor (7). Recently, interest has grown in the observations by several groups that circulatory monocytes express distinctive chemokine receptors, particularly with respect to CX3CR1 and CCR2 (also known as receptor for monocyte chemoattractant protein 1
[MCP-1]), as well as antigen markers such as Gr-1; CD14, a receptor for LPS-binding protein; and CD16, an Fc receptor $(8,9)$. Figure 1 summarizes the present consensus that different monocyte subsets give rise to constitutively present, resident macrophages and to tissue macrophages that have been recruited in increased numbers to local sites of infection and immunologic injury. There is evidence that this distinctive recruitment property of $\mathrm{CD} 14^{+} \mathrm{CD} 16^{+}$, which is not present in $\mathrm{CD} 14^{++} \mathrm{CD} 16^{-}$monocytes, mediated by CX3CR1 ${ }^{\text {hi }}$ CCR2-CD 62 ligand ${ }^{-}$(CX3CR1 $1^{\left.\text {hi CCR } 2-C D 62 L^{-}\right)}$and CX3CR $1{ }^{\text {lo } C C R} 2{ }^{+} \mathrm{CD} 62 \mathrm{~L}^{+}$subpopulations, respectively, is conserved in several species, including mice and humans. While the origin of microbially elicited, acute inflammatory macrophages is more readily demonstrable experimentally, the

\section{Table 1}

Modulation of macrophage phenotype

\begin{tabular}{|c|c|c|c|}
\hline Category & Stimulus & Selected marker changes & Function \\
\hline Innate activation & $\begin{array}{l}\text { Microbial products, e.g., LPS, } \\
\text { other TLR ligands }\end{array}$ & $\begin{array}{c}\text { Costimulatory molecule expression, } \\
\text { MARCO upregulation }\end{array}$ & Phagocytosis, adaptive immunity \\
\hline Classical activation & IFN- $\gamma$ & $\begin{array}{l}\text { MHCII upregulation, proinflammatory } \\
\text { cytokine secretion, iNOS2 }\end{array}$ & $\begin{array}{l}\text { Cell-mediated immunity, } \\
\text { e.g., intracellular pathogens }\end{array}$ \\
\hline Alternative activation & IL-4, IL-13 & $\begin{array}{l}\text { Upregulation of MHCII, arginase, mannose } \\
\text { receptor, Ym1, FIZZ1 (resistin-like); production } \\
\text { of selected chemokines; macrophage fusion }\end{array}$ & $\begin{array}{c}\text { Parasitic and allergic immunity, } \\
\text { repair }\end{array}$ \\
\hline $\begin{array}{l}\text { Innate and acquired } \\
\text { deactivation }\end{array}$ & $\begin{array}{c}\text { Apoptotic cells, IL-10, } \\
\text { glucocorticoids, TGF- } \beta, \text { PGE } 2\end{array}$ & $\begin{array}{l}\text { Various surface and secretory } \\
\text { markers, e.g., anti-TNF- } \alpha \text { actions }\end{array}$ & $\begin{array}{l}\text { Antiinflammatory effects, } \\
\text { altered immunity }\end{array}$ \\
\hline
\end{tabular}




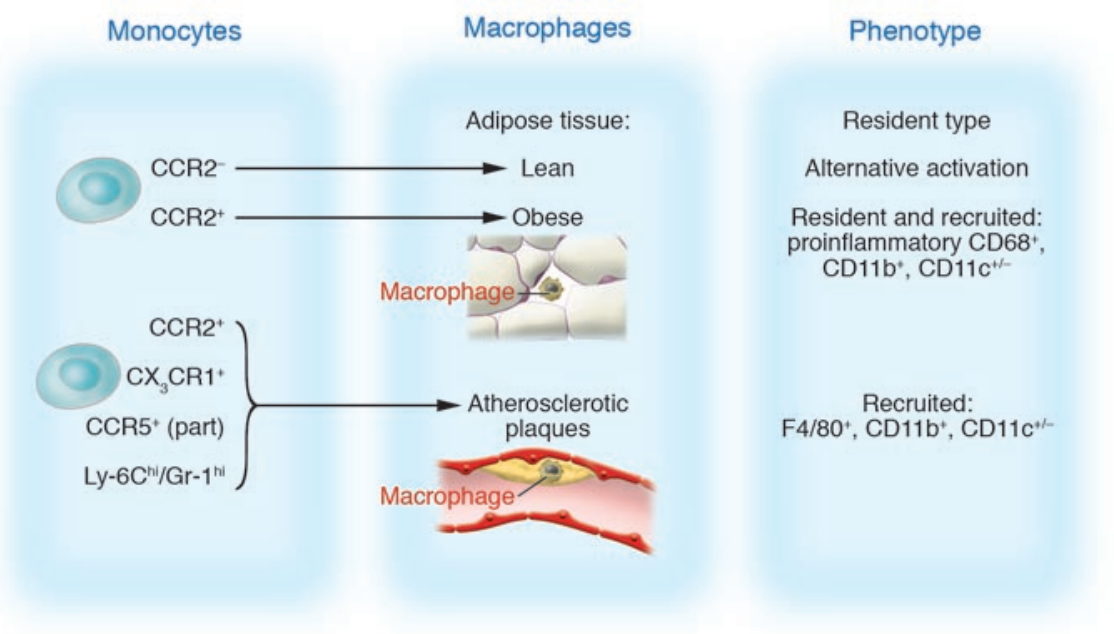

\section{Figure 2}

Monocyte and macrophage recruitment and response to tissue lipids. Summary of findings reported in the present studies (3-5). It is not clear whether individual monocytes express all markers and whether cells of each subset are recruited stochastically from the circulation. Some markers are not shown, which reflects lack of study rather than absence of expression. See text for role of $\mathrm{Gr}-1^{10}$ cells in atherosclerosis and in the generation of CD11 $\mathrm{c}^{-}$cells. CCR5 involvement is partial (part; see text).

sources of resident tissue macrophages, which turn over more slowly, are less clear. The origins of nonclassical inflammatory macrophages elicited by metabolic stimuli, such as modified (lipo)proteins in atherosclerosis, diabetes mellitus, and Alzheimer disease, have not been hitherto characterized.

Once present in tissues, mature macrophages exhibit another level of heterogeneity. Microbial stimuli acting through a variety of TLRs and non-TLRs, such as dectin-1, induce a range of transcriptional and nontranscriptional effector responses (known as innate activation) $(9,10)$. The cytokine IFN- $\gamma$ induces a signature of proinflammatory antimicrobial activities (known as classical activation) that are important in cell-mediated immunity to intracellular pathogens and are associated with Th1 lymphocyte responses. A distinctive, "alternative activation" profile is associated with Th2-type polarized responses. Characteristic features of IL-4and IL-13-induced (M2) phenotypes include enhanced MHC class II expression, as in IFN- $\gamma$-induced M1 macrophages, but distinctive upregulation of endocytic lectin-like receptors (e.g., the mannose receptor) and of arginase, rather than inducible NOS2 (Table 1). Alternative activation is strongly associated with extracellular parasitic infections, allergy, humoral immunity, and fibrosis. Macrophage deactivation is equally complex and characterized by distinctive, partially overlapping signatures of gene expression induced by IL-10, glucocorticoids, TGF- $\beta$, and other regulatory mediators.

A further level of heterogeneity to consider relates to the origin, differentiation, and activation of myeloid DCs (11). These cells arise from common progenitors and share many phenotypic characteristics with macrophages, namely phagocytosis, receptor expression, and cytokine secretion, but acquire a unique antigen-presenting capacity for naive $\mathrm{T}$ lymphocytes as they undergo a complex process of maturation. They become actively migratory cells, responding to selected chemokines as they deliver antigen to draining lymph nodes. However, DCs display considerable heterogeneity in lymphoid and nonlymphoid tissues, which has been difficult to establish in situ, rather than in the simpler in vitro model systems that are widely employed, and they express many apparently selective markers, such as CD11c, also expressed by tissue macrophages.

\section{Macrophage-lipid interactions}

The distinction drawn between innate immunity and lipid homeostasis is artificial and is breaking down. For example, TNF- $\alpha$, the prototypical proinflammatory cytokine that is critical for host defense, has direct effects on metabolic pathways including lipid metabolism, as originally described by Cerami and colleagues and termed cachectin (12). The scavenger receptors expressed mainly by macrophages and endothelia are implicated in innate recognition of microorganisms, as well as in foam cell formation and uptake of modified lipoproteins and of apoptotic cells (13). Circulating lipoproteins, their cellular receptors and lipid transfer proteins, play an important role in LPSinduced responses, TLR stimulation, activation of intracellular signaling pathways, especially of NF- $\mathrm{BB}$, and activation of gene expression. The roles of nuclear receptors such as PPAR $\gamma$ in inflammation and of lipid metabolites in the initiation and resolution of inflammation fall outside the scope of this commentary. Recently, interest has grown in the possible interactions between macrophages and adipocytes, involving cytokines such as leptin, adiponectin, and effects of these and other paracrine mediators on insulin responses and signaling.

\section{New experimental findings}

The study in this issue by Swirski et al. (3) utilized apoE-KO (apoE $\left.E^{-/}\right)$mice on a C57BL/ 6 background, fed a high-fat "Western" diet over a period of many weeks to induce an increase in atheromatous lesions throughout the aorta. The authors characterized monocyte numbers mainly in blood and spleen, as well as in bone marrow, and distinguished their phenotype from that of more mature 
splenic macrophages. (In rodents, the spleen is also a major hemopoietic organ). Hypercholesterolemia was associated with gradual monocytosis, with a doubling time of approximately 1 month, due to a selective increase in the Ly-6C-positive subset. Monocytosis was ascribed to increased survival and continued proliferation, in part due to $\mathrm{M}-\mathrm{CSF}$, as well as impaired conversion of Ly- $6 \mathrm{C}^{\text {hi }}$ to Ly- $6 \mathrm{C}^{\text {lo }}$ cells; monocytosis decreased upon statininduced reduction of cholesterol. Clodronate-laden liposomes were used to deplete monocytes and macrophages in vivo. The recovery of Ly- $6 C^{\text {lo }}$ monocyte numbers, presumably from Ly- $6 C^{\text {hi }}$ precursors, occurred over 5 days in animals on a control chow diet but was impaired in the hypercholesterolemic mice. Monocytes recovered from enzyme-digested aortas and analyzed by FACS showed preferential accumulation of Ly- $6 \mathrm{C}^{\text {hi }}$ cells, especially in more severe lesions, localized by immunocytochemistry. Experiments confirmed that Ly-6Chi monocytes adhered preferentially to TNF- $\alpha$-activated endothelium under laminar flow conditions in vitro, irrespective of diet or apoE deficiency. Adoptive transfer of CD45.2 monocytes to congenic CD45.1 mice demonstrated recruitment of Ly- $6 \mathrm{C}^{\text {hi }}$ monocytes to atherosclerotic aortas followed by local, rapid differentiation into macrophages; this was confirmed by labeling of splenic monocytes with [ $\left.{ }^{111} \mathrm{In}\right]$ oxine and localized by autoradiography. Transfer studies with CCR2-KO mice confirmed that CCR2 was required.

It is not clear whether the glycosylphosphatidylinositol-linked (GPI-linked) protein Ly-6C is a marker or functional receptor/ligand for an adhesin on endothelial cells and whether it or its human equivalent provides a potential target for inhibition of monocytosis and associated atherosclerosis. Future studies should establish whether $\mathrm{CD} 14^{++} \mathrm{CD} 16^{-}$monocytosis is an etiological factor in human disease.

Tacke et al. (4) used a similar apoE-/-plus-diet model and also observed monocytosis with increased frequency of Gr- $1^{\text {hi }}$ monocytes (their Gr-1 mAb reacts with an epitope on both Ly-6C and Ly-6G, expressed on granulocytes as well). They employed an i.v. labeling method to mark blood monocyte subsets by phagocytosis of $0.5-\mu \mathrm{m}$ latex particles. A portion of $\mathrm{Gr}-1^{\text {lo }}$ monocytes in apoE $\mathrm{E}^{-/-}$mice can be specifically and efficiently labeled for more than 5 days; Gr-1 ${ }^{\text {hi }}$ monocytes were labeled by a modification of this procedure, wherein monocytes are transiently depleted by clodronate-loaded liposomes prior to latex injection. Latex is then transferred to bone marrow, and labeled Gr- $1^{\text {hi }}$ monocytes appear in the circulation after 2 days, remaining Gr- $1^{\text {hi }}$ for 4 days before converting into Gr- $1^{\text {lo }}$ cells by 7 days. Normalization of numbers and cell clearance made it possible to compare the tracking of cells of each subset into lesions; the labeling procedure itself did not affect cellular recruitment. Though both subsets entered plaques, initial entry of Gr-1 $1^{\text {hi }}$ monocytes was approximately 20 -fold greater than that of Gr- $1^{\text {lo }}$ cells, after normalization for frequency of labeled cells. A fat-rich diet increased rates of recruitment of both subsets. Another observation was that a subpopulation of cells expressing CD68, a pan-macrophage/DC marker, was CD $11 c^{+}$and that Gr-1 $1^{\text {lo }}$ monocytes were particularly predisposed to become CD $11 \mathrm{c}^{+}$cells.

Further studies examined the role of chemokine receptors in recruitment. Surgical transfer of donor atherosclerotic aortic arches was used to take advantage of different knockout strains lacking CCR2 and CX3CR1. CCR2 was shown to be required (it also plays a role in monocyte exit from the bone marrow). Unexpectedly, Gr-1 ${ }^{\text {hi }}$ monocyte entry into plaques also depended on CX3CR1, and a potential ligand for this receptor, CX3CL1, was detected on endothelium overlying plaques. Xenogeneic in vitro experiments with HUVECs confirmed that Gr-1 $1^{\text {hi }}$ mouse monocytes required both CCR2 and CX3CR1 for in vitro migration. Finally, mAb depletion studies were used to implicate CCR5, in part, in monocyte migration to plaques.

The overall conclusion was that multiple chemokine receptors operate to control the migration of monocyte populations. This study identified a role for CX3CR1, not previously found in acute inflammatory models, giving rise to speculation that CX3CR1 may be selectively involved in atherosclerotic plaque recruitment, whereas CCR2 is more widely required in response to different types of inflammatory stimuli (4). This may account for the observation that polymorphism in human CX3CR1 leads to relative protection from cardiovascular disease (14).

In the third article, Lumeng et al. (5) restricted their studies to analysis of the macrophage phenotype in lean and adi- pose tissue of wild-type apoE ${ }^{+/+} \mathrm{C} 57 \mathrm{BL} / 6$ mice. Obesity and insulin resistance were induced in male mice, which were fed a high-fat diet consisting of $45 \%$ of calories derived from fat, starting at 8 weeks of age, for up to 20 weeks. Control mice were fed a standard diet consisting of $4.5 \%$ of calories derived from fat. Epididymal fat pads were extracted and the stromal vascular fraction isolated after removal of adipocytes by flotation. Digested preparations were analyzed by FACS, using F4/80 and CD 11 b to identify macrophages, which could be isolated by magnetic immunoaffinity-positive selection.

Adipose tissue from lean mice contained macrophages that expressed several markers of alternative activation, including IL-10 as well as enhanced levels of Ym1, arginase, and mannose receptor (5). By contrast, macrophages isolated from adipose tissue of obese mice exhibited a proinflammatory phenotype, overexpressing IL-6, NOS2, and CCR2; this chemokine receptor was required for diet-induced but not constitutive cell recruitment, as shown with CCR2-KO mice. In addition, some of the recruited macrophages expressed the CD11c antigen, although no further attempt was made in the present study to examine other possible DC markers. It was shown that IL-10, which was overexpressed in lean mice, protected adipocytes from TNF- $\alpha$-induced insulin resistance in vitro. Leptin-deficient $o b / o b$ mice showed a similar increase in $\mathrm{F} 4 / 80^{+} \mathrm{CD} 11 \mathrm{c}^{+} \mathrm{CD} 11 \mathrm{~b}^{+}$cells.

These studies are consistent with a role for CCR 2 in the diet-induced recruitment of monocytes that acquired a proinflammatory phenotype, gradually replacing a population with an alternative activation phenotype consisting of CCR2-independent macrophages that are slow to turn over. It is not clear which local stimuli might be responsible for either type of polarization, although lipid-derived metabolites are likely mediators of the proinflammatory phenotype. The nature and role of the CD11 $\mathrm{c}^{+}$cells also need to be investigated further. One limitation of these results is that ex vivo studies are not a precise reflection of cellular heterogeneity in vivo, although a limited immunocytochemical analysis was performed. This must be done with great care, since, in my own experience, adipose tissue is prone to stain nonspecifically with a range of mAbs. The potentially important surface and metabolic interac- 
tions among adipocytes, monocytes, macrophages, endothelial, and other stromal cells also deserve further investigation.

\section{Conclusion}

These intriguing studies have yielded further insights into the complexity of monocytes and tissue macrophages and the role of different chemokine receptors in constitutive and lipid-induced recruitment (summarized in Figure 2). It is clear that enhanced recruitment induced by metabolic and infectious inflammatory stimuli differs in important aspects that need better definition, in view of their importance in modified, often chronic inflammatory disease processes such as diabetes, as well as atherosclerosis. The nature of monocyte/macrophage heterogeneity is also difficult to assess due to the considerable plasticity of macrophages, their repertoire of receptors and extensive biosynthetic capacity. Furthermore, it is important not to overinterpret the expression of a single marker, such as CD11c, as indicative of DC differentiation. A panel of markers should be investigated, in situ if possible, and even then in relation to the complexity of macrophage differentiation and modulation in different tissue microenvironments. As to possible therapeutic exploitation, further investigation is required before different subsets of cells can be selectively, efficiently, and safely targeted. The availability of well-characterized experimental mouse models, as described in the articles under discussion, makes this an attractive goal. Their possible relevance to human disease poses further challenges for pathophysiological, translational research.

\section{Acknowledgments}

I thank my colleagues for the work done in my laboratory that contributed to this commentary. Research is supported by the Medical Research Council UK, the Wellcome Trust, the British Heart Foundation, and the Arthritis Research Campaign.

Address correspondence to: Siamon Gordon, Sir William Dunn School of Pathology, University of Oxford, South Parks Road, Oxford OX13RE, United Kingdom. Phone: 44-1865-275534; Fax: 44-1865-275515; E-mail: siamon.gordon@path.ox.ac.uk or christine.holt@path.ox.ac.uk.

1. Ross, R. 1999. Mechanisms of disease: atherosclerosis - an inflammatory disease. N. Engl. J. Med. 340:115-126.

2. Shoelson, S.E., Lee, J., and Goldfine, A.B. 2006. Inflammation and insulin resistance. J. Clin. Invest. 116:1793-1801. doi:10.1172/JCI29069.

3. Swirski, F.K., et al. 2007. Ly-6C hi monocytes dominate hypercholesterolemia-associated monocyto- sis and give rise to macrophages in atheromata. J. Clin. Invest. 117:195-205. doi:10.1172/JCI29950.

4. Tacke, F., et al. 2007. Monocyte subsets differentially employ CCR2, CCR5, and CX3CR1 to accumulate within atherosclerotic plaques. J. Clin. Invest. 117:185-194. doi:10.1172/JCI28549.

5. Lumeng, C.N., Bodzin, J.L., and Saltiel, A.R. 2007. Obesity induces a phenotypic switch in adipose tissue macrophage polarization. J. Clin. Invest. 117:175-184. doi:10.1172/JCI29881.

6. Glass, C.K., and Witztum, J.L. 2001. Atherosclerosis: the road ahead. Cell. 104:503-516.

7. Smith, J.D., et al. 1995. Decreased atherosclerosis in mice deficient in both macrophage colony-stimulating factor (op) and apolipoprotein E. Proc. Natl. Acad. Sci. U. S. A. 92:8264-8268.

8. Geissmann, F., Jung, S., and Littman, D.R. 2003. Blood monocytes consist of two principal subsets with distinct migratory properties. Immunity. 19:71-82.

9. Gordon, S., and Taylor, P.R. 2005. Monocyte and macrophage heterogeneity. Nat. Rev. Immunol. 5:953-964.

10. Taylor, P.R., et al. 2005. Macrophage receptors and immune recognition. Annu. Rev. Immunol. 23:901-944.

11. Wu, L., and Shortman, K. 2005. Heterogeneity of thymic dendritic cells. Semin. Immunol. 17:304-312.

12. Beutler, B., Mahoney, J., Le Trang, N., Pekala, P., and Cerami, A. 1985. Purification of cachectin, a lipoprotein lipase-suppressing hormone secreted by endotoxin-induced RAW 264.7 cells. J. Exp. Med. 161:984-995.

13. Peiser, L., Mukhopadhyay, S., and Gordon, S. 2002. Scavenger receptors in innate immunity. Curr. Opin. Immunol. 14:123-128.

14. McDermott, D.H., et al. 2003. Chemokine receptor mutant CX3CR1-M280 has impaired adhesive function and correlates with protection from cardiovascular disease in humans. J. Clin. Invest. 111:1241-1250. doi:10.1172/JCI200316790. 


\title{
Atherogenic remnant lipoproteins: role for proteoglycans in trapping, transferring, and internalizing
}

\author{
Robert W. Mahley1,2,3,4,5 and Yadong Huang1,2,3,6 \\ ${ }^{1}$ Gladstone Institute of Neurological Disease and ${ }^{2}$ Gladstone Institute of Cardiovascular Disease, San Francisco, California, USA. ${ }^{3}$ Department of Pathology, \\ ${ }^{4}$ Department of Medicine, ${ }^{5}$ Cardiovascular Research Institute, and ${ }^{6}$ Department of Neurology, UCSF, San Francisco, California, USA.
}

\begin{abstract}
Unraveling the mechanisms controlling remnant lipoprotein clearance is important, as these lipoproteins are highly atherogenic. The most critical molecule in this process is apoE, which mediates high-affinity binding of remnant lipoproteins to members of the LDL receptor (LDLR) family and cell-surface heparan sulfate proteoglycans (HSPGs), which have been shown to play major independent as well as cooperative roles in remnant lipoprotein clearance. While all the players may have been identified, our understanding of how they interact and function together continues to evolve. In this issue of the JCI, MacArthur et al. (see the related article beginning on page 153) demonstrated that HSPGs under normal physiological conditions are critically important in the clearance of remnant lipoproteins, independent of LDLR family members. The complexity of VLDL and chylomicron remnant clearance was exemplified by the studies of Jones et al., also in this issue (see the related article beginning on page 165). Despite defective clearance of LDL in mice with a deficiency in the adaptor protein controlling internalization of the LDLR, called autosomal recessive hypercholesterolemia (ARH), remnant lipoprotein clearance was not grossly abnormal. A likely explanation is that the abnormal LDLRs bind the remnants and then transfer them to another acceptor for internalization. While the studies clearly demonstrate that the LDLR-related protein 1 is not involved and suggest a role for an additional unidentified receptor, it remains a possibility that HSPGs are responsible for remnant uptake by hepatocytes in the presence of defective LDLR internalization.
\end{abstract}

\section{Remnant lipoproteins from the liver and intestine}

Remnant lipoproteins are cholesterol-rich particles that are generated during circulation by lipolytic processing of liver-synthesized VLDL and intestine-synthesized chylomicrons $(1,2)$. Remnants are extremely atherogenic lipoproteins $(3,4)$. Lipoprotein lipase (LPL) on endothelial cell surfaces in capillaries and, to a lesser extent, hepatic lipase (HL) in the liver hydrolyze the triglycerides of these particles and generate remnants that under normal

Nonstandard abbreviations used: ARH, autosomal recessive hypercholesterolemia; FH, familial hypercholesterolemia; GlcNAc, N-acetyl glucosamine; HL, hepatic lipase; HLP, hyperlipoproteinemia; HSPG, heparan sulfate proteoglycan; LDLR, LDL receptor; LPL, lipoprotein lipase; LRP, LDLR-related protein; Ndst1, GlcNAc $N$-deacetylase/ $N$-sulfotransferase 1.

Conflict of interest: The authors have declared that no conflict of interest exists.

Citation for this article: J. Clin. Invest. 117:94-98 (2007). doi:10.1172/JCI30889. circumstances are rapidly and efficiently cleared from the blood by hepatocytes, where they are catabolized. Lipoproteins (density < 1.006) induced by cholesterol feeding in animals or occurring in patients with type III hyperlipoproteinemia (HLP, a genetic disorder characterized by remnant lipoprotein accumulation in the blood), called $\beta$-VLDL, are a mixture of both VLDL and chylomicron remnants, and their accumulation results in impairment of remnant clearance $(1,2,4)$. The major components of remnants, including $\beta$-VLDL, are apoB100, apoB48, apoE, and the apoCs. ApoE is the critical ligand responsible for remnant lipoprotein clearance $(1,5)$. LPL and HL may also serve as ligands under some circumstances (6-9).

\section{Role of apoE in remnant binding and uptake}

Some of the first evidence defining a critical role for apoE in remnant clearance involved i.v. infusion of apoE in cholesterol-fed rabbits and the demonstration of accelerated clearance of $\beta$-VLDL remnants from the blood and their uptake by the liver (10). In transgenic mice and rabbits, overexpression of apoE accelerated remnant clearance (11-13). Furthermore, apoE was localized to the sinusoids in the space of Disse, and after infusion of remnant lipoproteins, apoE disappeared from the sinusoids and appeared in hepatocytes (14). ApoE-knockout mice have markedly defective remnant lipoprotein clearance $(15,16)$. Clearly, LPL and HL do not substitute for apoE in remnant clearance.

Compelling proof of apoE's role as a critical ligand for remnant lipoproteins came from understanding type III $\operatorname{HLP}(2,4)$. Type III HLP is associated with genetic mutations in apoE. The most common apoE variant [apoE2(R158C)] acts as a susceptibility factor that requires a second hit (obesity, hypothyroidism, menopause, etc.) to induce remnant lipoprotein accumulation $(2,4)$. The majority of apoE2 homozygotes actually have normal or even low plasma lipid levels and no significant impairment of remnant clearance. This apoE variant is also defective in binding to the LDL receptor (LDLR), the LDLR-related protein (LRP), and heparan sulfate proteoglycans (HSPGs). A transgenic animal model expressing this apoE variant can be induced to develop type III HLP (17).

A variant in which arginine at residue 142 is replaced by a cysteine [apoE(R142C)] has a dominant mode of type III HLP transmission with marked remnant lipoprotein accumulation and premature atherosclerosis (a second hit is not required) (18). Expression of this variant in transgenic mice causes remnant accumulation (19). This variant is defective in binding to LDLR, LRP, and HSPGs $(2,20)$.

However, critical information learned from studies of these apoE variants not only supports the importance of apoE 